\title{
La enseñanza emocional en el aula invertida: promover valores viendo cortometrajes
}

Ma Montserrat Castro Rodríguez. Universidad de A Coruña

0000-0002-5544-4421

Óscar Mallón Suárez. Universidad de A Coruña

0000-0002-8090-7715

Recepción: 14/10/2019 | Aceptado: 24/10/2019

Correspondencia a través de ORCID: Ma Montserrat Castro

iD 0000-0002-5544-4421

Citar: Castro, MM y Mallón, O (2019). La enseñanza emocional en el aula invertida: promover valores viendo cortometrajes. REIDOCREA, 8(2), 86-102.

Resumen: El desempeño de la función docente conlleva integrar contenidos y objetivos curriculares a través de secuencias didácticas y diferentes metodologías, utilizando recursos y materiales que puedan provocar el interés en el aprendizaje por parte del alumnado. En este artículo se presentan los resultados de una investigación que desarrolla un maestro principiante, que planifica sus propias secuencias didácticas, para trabajar contenidos de educación emocional en la etapa de Educación Primaria, usando la metodología flipped classroom. El docente selecciona y analiza los materiales, en este caso, cortometrajes. Los objetivos de su investigación son identificar, analizar y seleccionar los cortometrajes adecuados para su integración en el aula. La muestra final es de 30 cortometrajes. Se usa una metodología mixta para el análisis documental, haciendo uso de dos instrumentos de recogida de información: la escala auto-aplicada de identificación con los personajes de formatos audiovisuales (EDI) y una ficha de elaboración propia para cada material. Los resultados obtenidos corroboran la existencia de abundantes cortometrajes, de fácil manejo y comprensión, adecuados para el trabajo de la educación emocional utilizando el método del aula invertida. Destaca la desigual disponibilidad de cortometrajes en función de las temáticas abordadas (acoso escolar, violencia de género, empatía y valores). Se ha constatado que la variedad de los cortometrajes existentes facilita la adaptación a las necesidades educativas individuales y colectivas.

Palabras clave: Materiales | Educación emocional

The emotional education through the flipped classroom: promoting values watching short films

Abstract: The achievement of the teacher training entails the inclusion of contents and curricular objectives through different didactic sequences, using resources and materials to cause the interest to learn in the student body. In this article, there are several results of research that an inexperienced teacher develops to plan his own didactic sequences and work contents of emotional education in the primary education level, using the methodology flipped classroom. The teacher selects and analyses the materials, in this case, short films. The objectives of his investigation are: identify, analyse and select the appropriate short films to use in the classroom. The final sample are 30 short films. It was used a mixed methodology for the documentary analysis, using two instruments to gather the information: a self-report scale that measures the empathy and identification with characters of audiovisual fictional stories (EDI) and an own file card to each material. The results show the existence of multiple short films, easy to understand and manage, adapted to work the emotional education using the flipped classroom methodology. It stands out the unequal availability of the short films taking into account the selected topics. It has been confirmed that the variety of the present short films facilitate the adaptation of the educative needs individually and collectively.

Key words: Materials | Emotional education

\section{Introducción}

Muchos de los problemas actuales que encuentra el alumnado en la escuela no están relacionados con su capacidad intelectual. Más bien, es su "analfabetismo emocional" el causante de conductas disruptivas (Fariña, Arce y Novo, 2008), de dificultades asociadas al fracaso escolar (Orón, 2016) y del abandono de los estudios a temprana edad (Álvarez y Martínez, 2016). En este contexto, se justifica la necesidad de integrar de forma transversal en las escuelas la educación emocional. 
En este artículo, un docente principiante realiza un proceso de investigación en torno a qué y cómo seleccionar materiales que le pueden ayudar a planificar su práctica educativa usando la metodología flipped classroom para trabajar la educación emocional en el aula de Educación Primaria (EP). Para el desarrollo de su trabajo utiliza la escala auto-aplicada de identificación con los personajes de formatos audiovisuales (en adelante EDI) y una guía de análisis de elaboración propia. Su objetivo es analizar los materiales, para luego seleccionar aquellos que le puedan resultar más adecuados a sus objetivos. Paralelamente quiere experimentar los instrumentos (cortometrajes) con el fin de valorar su idoneidad con el alumnado.

\section{La educación emocional en Educación Primaria}

La inteligencia emocional fue definida por Mayer y Salovey (1997) haciendo referencia a un tipo de inteligencia que tendría que aunar cinco capacidades: el conocimiento de las emociones propias, el control emocional, la capacidad para auto-motivarse, la empatía y las habilidades sociales. Guarda estrecha relación con la inteligencia interpersonal, una de las ocho inteligencias de la Teoría de las Inteligencias Múltiples definida por Howard Gardner.

Actualmente el profesorado se encuentra con determinados obstáculos que pueden interferir a la hora de trabajar en el aula y que es necesario abordar para mejorar los procesos de enseñanza y aprendizaje. Wirawan, Tamar y Bellani (2019) destacan la escasa motivación en el aula que presenta un alto porcentaje del alumnado, su falta de interés, atención, así como un déficit en su participación y cooperación. Torres (2012) alude a un aumento progresivo de las conductas disruptivas, posiblemente en relación con metodologías obsoletas, el impacto de las TIC, la globalización y los flujos migratorios. En este sentido, Mestre et al. (2006) atribuyen a las habilidades emocionales la posibilidad de contribuir a una mejor adaptación social y académica de los discentes, posibilitando la mejora de las condiciones para trabajar en el aula, facilitando su autorregulación y un mejor uso de sus emociones para desarrollar el pensamiento a través de la concentración, desembocando en respuestas afectivas a situaciones de estrés, a relaciones "armoniosas" en el aula y a la adaptación social y el rendimiento académico como ejes para la implicación en el trabajo y en las relaciones con sus iguales.

El reconocimiento de la importancia de la inteligencia emocional en la construcción personal, conlleva su puesta en valor dentro del proceso de enseñanza y aprendizaje ya desde edades muy tempranas (Bueno i Torrens, 2017; Sepúlveda, MayorgaFernández y Lacal, 2019). En este contexto, surge la necesidad de reflexionar sobre cómo introducirlo en la escuela: el valor pedagógico de considerar la inteligencia emocional y la educación emocional (Del Carmen y Portillo-Peñuelas, 2018; Sadipour, Ghavam, Farrokhi, Assadzadeh y Sameti, 2019), el uso de metodologías, de recursos y materiales didácticos (Chen y Guo, 2018), qué cómo y para qué evaluar (Rodrigo-Ruiz, Cejudo y Pérez, 2019), qué agentes deben participar, factores que intervienen en el desarrollo de la inteligencia emocional (Alonso Ferres, Berrocal de Luna y Jiménez Sánchez, 2018; Alam y Ahmad, 2018; Pulido-Acosta y Herrera-Clavero, 2019; Hu, Shang y Xia, 2019).

\section{La flipped classroom para la educación emocional}

Para Aguilera et al. (2017), la flipped classroom, se presenta como una alternativa a la caduca clase tradicional, muy poco atractiva para el alumnado, porque le atribuye un rol pasivo, inhibiendo la interactividad en el aula. Según Sola et al. (2019), esta metodología, más actual, entiende que el discente debe ser un agente activo en su 
aprendizaje. Tourón, Santiago y Díez (2014) consideran que la clase invertida combina el trabajo dentro y fuera del aula, con el objetivo de poner en juego distintos procesos y experiencias de aprendizaje para el desarrollo de la práctica docente. Cada estudiante asume un rol activo en el proceso, mientras que el profesorado actuará como guía y orientador en la búsqueda del conocimiento. El aula se convierte en el lugar donde se analicen las dudas, se compartan ideas y experiencias, se realicen actividades que promuevan el diálogo, se puedan extraer conclusiones y el intercambio de opiniones para ir construyendo un conocimiento propio a través de la interacción con el resto del aula.

Pero la implementación de una metodología no puede llevarse a cabo sin analizar previamente las ventajas/desventajas de su uso, teniendo como objetivo el evitar sus inconvenientes y aprovechar sus posibles potencialidades. Desde nuestro punto de vista, las principales ventajas y desventajas son las siguientes:

\begin{tabular}{|c|c|}
\hline \multicolumn{2}{|c|}{ La metodología de la aula invertida (flipped classroom) } \\
\hline VENTAJAS & INCONVENIENTES \\
\hline Incrementa la motivación del alumnado & Aumenta la brecha digital \\
\hline $\begin{array}{c}\text { Atención personalizada del docente a la } \\
\text { diversidad }\end{array}$ & Discrimina al alumnado y a familias con pocos recursos \\
\hline $\begin{array}{c}\text { El docente comparte la información con la } \\
\text { comunidad educativa }\end{array}$ & Aumenta el tiempo ante una pantalla. \\
\hline $\begin{array}{l}\text { Fomenta el trabajo colaborativo y/o cooperativa } \\
\text { en el aula }\end{array}$ & $\begin{array}{c}\text { Es necesario que los docentes tengan buena formación } \\
\text { en el uso de las TIC }\end{array}$ \\
\hline $\begin{array}{c}\text { Se accede a los contenidos cuando se quiere y } \\
\text { las veces que se desee }\end{array}$ & $\begin{array}{l}\text { Para tener éxito, los alumnos y las alumnas deben } \\
\text { implicarse porque si no las dudas no se resuelven }\end{array}$ \\
\hline $\begin{array}{c}\text { Fomenta la creatividad y el pensamiento crítico } \\
\text { entre el alumnado }\end{array}$ & $\begin{array}{c}\text { No todo el alumnado tiene la autonomía o la capacidad } \\
\text { para aprender a través de vídeos }\end{array}$ \\
\hline Implicación de la comunidad & Desigualdad de oportunidades \\
\hline
\end{tabular}

Evidentemente, las ventajas de esta metodología son coherentes con las exigencias de un modelo de escuela que pretende incluir a todo el alumnado: motivación, contextualización, personalización, colaboración/cooperación, flexibilidad en el acceso al conocimiento, creatividad, pensamiento crítico. Según Parrilla y Moriña (2006), el aprendizaje cooperativo, flexible, la participación activa del alumnado y la organización social del aula resultan claves para desarrollar una práctica educativa inclusiva. Uno de los aspectos más valorados en las investigaciones sobre las aportaciones de la metodología del aula invertida es su potencial para el trabajo cooperativo (González y Carrillo, 2016)

Sin embargo, existen otras cuestiones en esta metodología que es necesario analizar, actuando con precaución, con el objetivo de llevar a cabo iniciativas que garanticen la igualdad de oportunidades. Otros aspectos a considerar son: trabajar con los tiempos necesarios o elaborar un horario previo de trabajo antes del visionado; informar y formar a las familias con el objetivo de que puedan realizar la actividad en igualdad de oportunidades como, por ejemplo, proporcionar los recursos y materiales para cada actividad; formar a todo el alumnado en las competencias digitales necesarias para usar 
los dispositivos, construyendo un espacio de diálogo con la familia para evitar miedos, ayudando a extraer sus potenciales; proporcionar los espacios y tiempos adecuados.

\section{Cortometrajes en la metodología flipped classroom para trabajar la educación emocional.}

Un cortometraje es una expresión artística audiovisual que según la Real Academia de la Lengua se define como "película de corta e imprecisa duración" (2016) y de acuerdo con el Diccionario Técnico Akal de Cine (Konisgberg, 2004) lo identifica como "cualquier filme de tres rollos o menos y de duración inferior a 30 minutos".

Teniendo en cuenta sus características, el cortometraje es un recurso de especial interés para trabajar con estudiantes en la etapa de educación primaria, porque sus características pueden coincidir con los gustos e intereses del alumnado. Su corta duración focaliza su atención. Los niños y las niñas están acostumbrados a ver televisión, cine u otros recursos digitales en su tiempo libre. Como afirma Méndez (2001:23), los medios de comunicación, y en particular el cine, "actúan como educadores informales", que no sólo contribuyen a la formación, sino también a una actitud crítica, predisposición al aprendizaje, etc. En nuestras experiencias docentes, se ha identificado el gran potencial de las imágenes animadas, que se proyectan en formatos familiares y que se asocian a contenidos que son del interés del alumnado. El cine es un medio muy eficaz para enseñar valores y contravalores, porque como argumenta Pereira (2005), proporciona la oportunidad de debatir críticamente temáticas que responden a la vida, a su día a día.

Experiencias como las de Marín y González (2006), González y Carrillo (2016) constatan cómo el cine puede actuar como recurso didáctico y pedagógico al servicio de la enseñanza. En los últimos años se ha visto la metodología flipped classroom como una oportunidad para el trabajo de las competencias digitales desde la perspectiva de atención a la diversidad (Dafonte, Corbacho y Miguéz, 2017). Desaia, Jabeen, Abdul y Raoa (2018) consideran muy apropiado el uso de películas a través de la flipped classroom, especialmente para el conocimiento de otras civilizaciones y culturas.

\section{Objetivos o hipótesis}

Los objetivos de esta investigación son conocer los resultados de la evaluación que un docente principiante de educación primaria extrae después de identificar, analizar y seleccionar cortometrajes que va a utilizar en su clase para trabajar la educación emocional a través de la metodología flipped classroom

A partir de estos objetivos se formulan tres preguntas: ¿Es posible trabajar la educación emocional con este cortometraje?, ¿Qué aspectos positivos se aprenden?, ¿Se puede transferir lo aprendido a la vida diaria?

\section{Método}

Esta investigación presenta los resultados del proceso que sigue un docente principiante para la selección de material audio-visual (cortometrajes) que utilizará en su clase para trabajar contenidos relacionados con educación emocional, utilizando la metodología flipped classroom. El método de investigación es mixto, integra datos e información de carácter cualitativo y cuantitativo. El docente a medida que observa los cortometrajes, cumplimenta la escala EDI elaborada por Igartua y Páez (1998) y recoge datos cuantitativos y una ficha de elaboración propia que recoge información sobre cada uno de los cortos. 
Los resultados obtenidos no tienen intención de generalización, sino de expresar un resultado derivado del análisis de los cortometrajes que hace el propio docente para seleccionar el material de acuerdo con sus propios intereses profesionales, teniendo en cuenta los objetivos que pretende y el contexto de desarrollo de su aula.

\section{Muestra}

La muestra consta de 30 cortometrajes seleccionados en la página web www.educaciontrespuntocero.com y el referente web youtube, de un total de 60 analizados. Los criterios de selección de la muestra fueron:

- La calidad y valor educativo de Pixar, referente mundial en el mundo de la animación.

- La variación del contenido de los cortometrajes propone temáticas que muestran problemas cercanos o conocidos por los niños y niñas y que justifican la diversidad como eje vertebrador de la escuela inclusiva. Esta selección de las temáticas y valores a trabajar se realizó en concordancia con la Programación General Anual del Centro.

- Que abordaran contenidos incluidos en el currículum de Educación Primaria.

- Duración no superior a 8 minutos.

\begin{tabular}{|llll|}
\hline & & Tabla 2. Muestra seleccionada. \\
\hline 1. & Un conte & 2. & Deadlines \\
3. & El maltrato sutil & 4. & ¿Te atreves a soñar? \\
5. & El sándwich de Mariana & 6. & Dollface \\
7. & Silencios & 8. & Slimtime \\
9. & Broken Wings & 10. & El piano \\
11. & Cuerdas & 12. & Piper \\
13. & El regalo & 14. & The lost thing \\
15. & El cazo de Lorenzo & 16. & Take me home \\
17. & En tus brazos & 18. & The moon \\
19. & Mi hermanito de la luna & 20. & El viaje de Said \\
21. & Por 4 esquinitas de nada & 22. & For the birds \\
23. & Tamara & 24. & EMI \\
25. & A Cloudy lesson & 26. & Idiots \\
27. & A bolboreta Sabela & 28. & Pipas \\
29. & Changing batteries & 30. & Reach \\
\hline
\end{tabular}

\section{Instrumento}

La Escala EDI es una herramienta usada muy frecuentemente tanto en la investigación como en el proceso de evaluación de cortometrajes en el aula (Igartúa y Páez ,1998). 


\begin{tabular}{|l|}
\multicolumn{1}{|c|}{ Tabla 3. Ficha de análisis de información de cada cortometraje. } \\
\hline Título \\
Trama \\
Personajes \\
Música \\
Colores \\
Valores que trabaja \\
Conclusión \\
Escala EDI (MEDIDA EMPATÍA) \\
\hline
\end{tabular}

\section{Procedimiento}

Inicialmente se procedió al visionado de 60 cortometrajes, del que finalmente se seleccionaron 30 (animación y no animación) de diferentes temáticas que desarrollan problemas cercanos o conocidos por los niños en su etapa educativa (acoso escolar, violencia de género, inmigración, etc.), así como, la gestión de las emociones primarias (miedo, ira, tristeza o enfado).

Posteriormente se procedió al análisis de cada uno de los cortometrajes utilizando el EDI y la ficha con el vaciado de contenido.

Con los datos e información obtenidos se procedió a su análisis e interpretación con la ayuda del Excel 2013.

\section{Resultados}

\section{Distribución de los cortometrajes según las temáticas abordadas}

En los resultados obtenidos en la muestra, se detecta una distribución desigual de acuerdo con los contenidos. Abundan los cortos que abordan temáticas más generales, frente a temáticas tan específicas como la violencia de género y el acoso escolar. Algunas causas que lo justifican es la limitación de la selección realizada en las fuentes originarias; también la dificultad de abordar ciertos contenidos para edades tan tempranas, entre otros.

\begin{tabular}{|c|c|}
\hline \multicolumn{2}{|c|}{ Tabla 4. Temática de los cortometrajes } \\
\hline Violencia de género & 1,2 \\
Acoso escolar & 3,4 \\
& $5,6,7,8,9,10,11,12$ \\
Diversidad /Empatía & $13,14,15,16,17,18,19,20,21,22,23,24,25$ \\
Valores & $26,27,28,2930$ \\
\hline
\end{tabular}




\section{Resultados de la escala EDI y la ficha de análisis}

La figura 1 presenta las medias aritméticas obtenidas en cada uno de los ítems de la escala EDI. Como puede constatarse, en general, es positiva, teniendo en cuenta que la mayoría de los cortometrajes obtienen una valoración de aceptación de buena o bastante buena: 6 de los ítems alcanzan una media de 4 o superior, lo que indica una valoración de "bastante" o "muy buena"; 9 en el intervalo de 3 hasta 3,99, coincidentes con una valoración regular tirando a bastante; tan sólo dos de ellos no superan la puntuación media de 1. Las valoraciones negativas de algunos negativos pueden estar condicionadas por la experiencia personal del sujeto que visionó la muestra. Algunos cortometrajes presentan personajes o situaciones con las que no se identificó, no comprendió la temática representada o su reacción fue de indiferencia. De manera global, casi todos los cortometrajes analizados cumplen con los criterios asignados por la escala EDI, aportando fiabilidad y validez a la muestra como recurso pedagógico a tener en cuenta para trabajar valores y educación emocional en el aula.

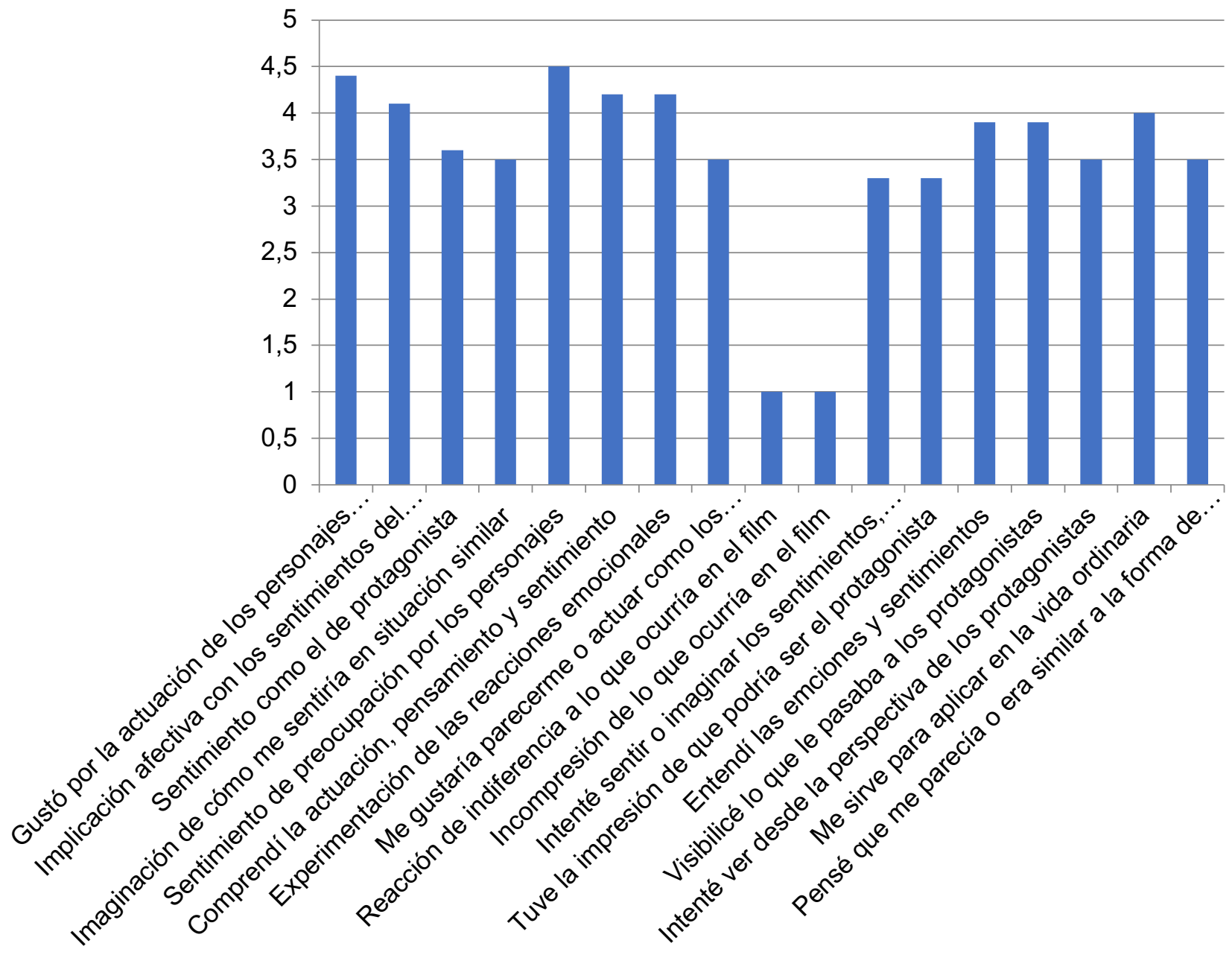

Figura 1. Medias aritméticas de los ítems de la Escala EDI.

A continuación se presentan las valoraciones de cada uno de los ítems de la escala, complementando las justificaciones con las informaciones extraídas de las fichas de análisis. 
30
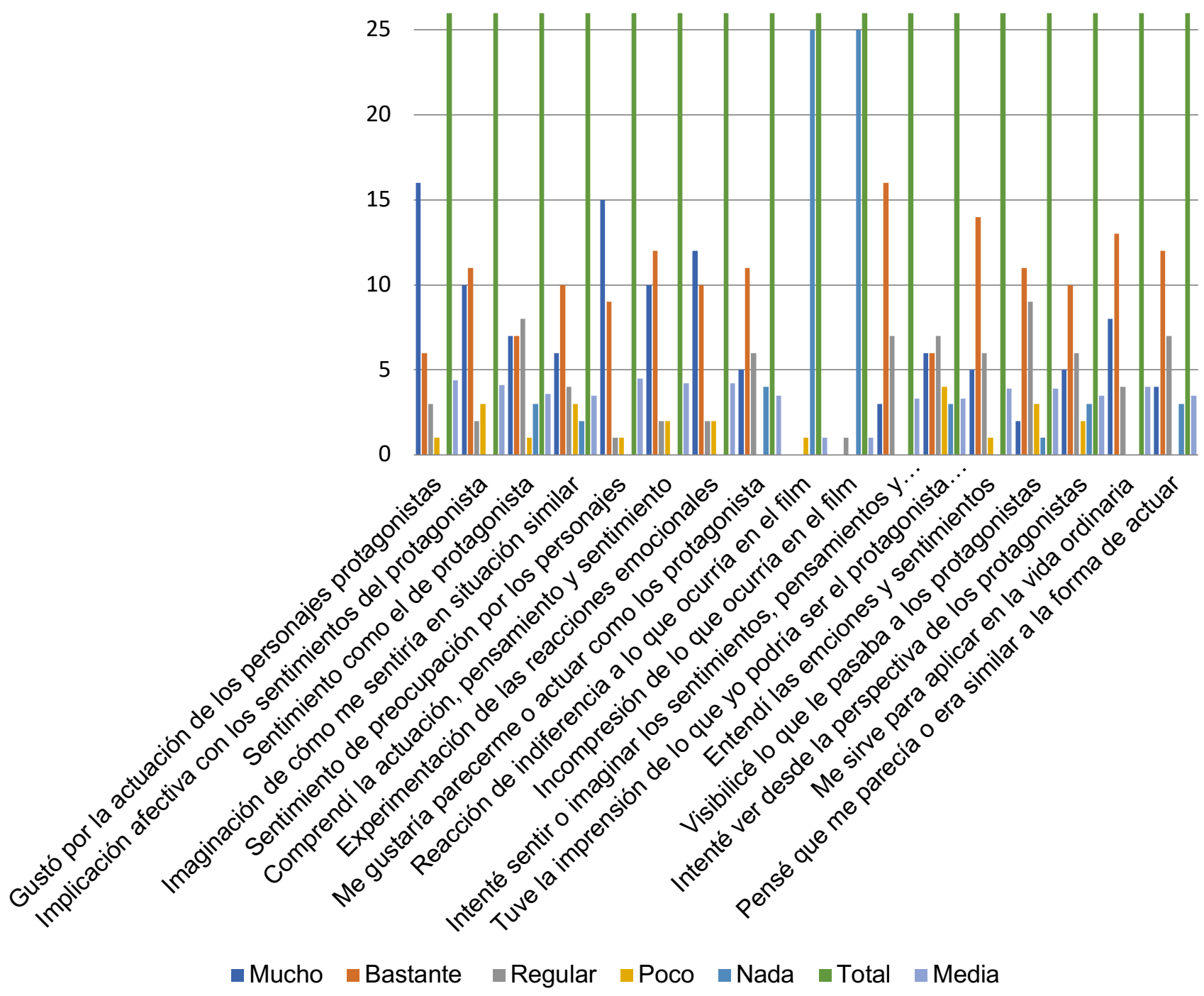

Figura 2. Distribución en porcentajes de los ítems de la Escala EDI.

En la figura 1, se presentan los resultados de cada ítem de acuerdo con las valoraciones de una escala de likert. Esta gráfica que viene a detallar la distribución de la muestra en cuanto a los porcentajes alcanzados por cada una de las categorías de la escala.

- Actuación de los personajes

La mayoría de los cortometrajes alcanzaron una puntuación positiva; la media refleja un 4,5 lo que sitúa la puntuación entre "bastante" y "mucho", exponiendo que, por lo menos 26 cortometrajes contienen personajes cuya actuación resultó del agrado del espectador. "Maltrato sutil" y "El sándwich de Mariana" son los cortometrajes que registraron las puntuaciones más bajas. En las valoraciones de la ficha se incluyen el reflejo de conductas desagradables o entendidas como contrarias a la convivencia (violencia de género, acoso escolar). 
- Sentimientos de los protagonistas.

Los sentimientos que emanan de las actuaciones de los protagonistas implican afectivamente al espectador, identificándose con lo que sienten, sufren o viven en los cortometrajes. La mayoría de los cortos consiguen captar al evaluador (4=bastante), frente a un número reducido de la muestra que refleja sentimientos que no son agradables ni se pueden considerar como "positivos" para poder empatizar con ellos como se argumenta en las valoraciones cualitativas. Son, como en el caso anterior los cortos "Maltrato sutil", "El sándwich de Mariana", "Un comte" y "Silencios".

- Empatía con los personajes y afinidad con los protagonistas:

Como reflejan las gráficas 1 y 2 , el evaluador empatizó con una amplia mayoría de los personajes, con puntuaciones entre 4 y 5 . De nuevo las temáticas más desagradables propiciaron puntuaciones más bajas, donde se argumenta la falta de experiencia de las situaciones reflejadas en las películas.

- Preocupación por lo que le pasa a los personajes.

En el $53 \%$ de la muestra que obtiene puntuaciones entre 4 y 5 , pues consiguen que el evaluador sienta preocupación por lo que les pasa; sólo hay un cortometraje que registra una puntuación media $(p o c o=3)$ y otro en el que la puntuación es de "muy poco=2" por ser un cortometraje de animación cómico en el que el devenir de los personajes no causó preocupación al espectador de la muestra ("The birds").

- Comprender la actuación, pensamiento y sentimiento de los protagonistas

El $85 \%$ de la muestra alcanzó puntuaciones de 4 y 5 a excepción de "Maltrato sutil", "El sándwich de Mariana", "Silencios" "Un comte". La justificación es la dificultad que tuvo el evaluador para entender el entramado general de cada uno de ellos.

- Experimentar reacciones emocionales de los personajes.

EL $73 \%$ de la muestra recibe de nuevo puntuaciones muy altas (4 y 5), siendo el resto puntuado de forma más negativa, correspondiendo de nuevo a los temas más controvertidos, por su dificultad en la compresión y por la complejidad de los temas abordados, que quizás no se ajustan a los posibles destinatarios del proyecto.

- Parecerse o actuar como los protagonistas.

Al tener temáticas positivas, que trabajan emociones de manera asertiva, el espectador desearía parecerse o actuar como los personajes en todos los cortometrajes (73\% del muestreo, puntuaciones (4 y 5 ) con la excepción de los cuatro primeros de la lista, de temática controvertida.

- Comprensión del film

Es destacable que ninguno de los cortometrajes dejó indiferente al evaluador. Todos ellos son capaces de generar reacciones positivas o negativas según el caso.

- Traslación de los sentimientos, pensamientos y reacciones de los protagonistas. 
En este ítem ganan claramente las puntuaciones más elevadas (4 y 5). En las puntuaciones más bajas, la descripción del ítem no clarifica si hay que sentir o imaginar las reacciones, sentimientos y pensamientos de los protagonistas en el momento de la acción o antes del visionado, por lo que, en esos cortometrajes resulta difícil imaginar los sentimientos de los protagonistas por parte del espectador.

- Tener la impresión de ser el protagonista de la historia.

El maestro que realizó esta investigación no sintió la impresión de ser el protagonista en la mayoría de los cortometrajes. Un $46 \%$ de la muestra obtuvo puntuaciones entre 4 y 5. Esto sucede porque, salvo en los cortos cuya trama contiene la figura de una persona que enseña o ayuda o en aquellos en los que el aprendizaje tiene un peso relevante, el resto contempla una ficción que no se corresponde con nada de lo que hubiera podido vivir o experimentar.

- Entender las emociones y sentimientos de los protagonistas.

"El sándwich de Mariana", junto con "Silencios" y "Maltrato sutil" forman un conjunto de películas cuya temática aborda el problema del acoso escolar y de la violencia de género $y$, de toda la muestra, son las valoradas con puntuaciones más negativas (2=muy poco o 1=nada). Eso es significativamente positivo porque se rechazan esos malos comportamientos y no se aceptan las conductas mostradas en estos cortometrajes.

- Anticiparse a lo que le iba a pasar a los protagonistas.

"El sándwich de Mariana" cuya historia da un giro argumental que hace difícil anticiparse a lo que le pasa a los protagonistas; el resto de la muestra (99\%) contiene comportamientos por parte de los protagonistas que son predecibles (puntuaciones entre 4 y 5$)$.

- Intentar ver las cosas desde el punto de vista de los protagonistas y aplicación en la vida diaria

De nuevo las puntuaciones más altas superan a las más bajas siendo significativo las tramas de aquellos cortos que no parecen ser relevantes para el espectador por no haber vivido situaciones similares ( $57 \%$ de la muestra, puntuaciones entre 4 y 5 ). Su aplicación en la vida diaria es factible por ser un docente que busca un recurso significativo que ayude al alumnado a identificar sus emociones y mostrar empatía con los demás.

- El espectador se parecía o era muy similar en la forma de actuar del protagonista.

Existe un alto porcentaje de la muestra ( $65 \%$ con puntuaciones entre 5 y 4$)$ que reafirma la empatía por sus protagonistas al pensar que se podría actuar o ser parecido en la forma de actuar de los personajes.

\section{Discusión}

La evaluación del material realizada por el profesor le lleva a tomar la decisión de cuáles son los materiales más adecuados para el alumnado. Además de las puntuaciones que le atribuye a cada uno de los ítems para cada cortometraje, son especialmente interesantes las observaciones y valoraciones que aporta en la guía de análisis documental. Con frecuencia, justifica sus valoraciones en función de sus creencias, 
expectativas con respecto a las reacciones del alumnado o sus propias experiencias vividas. Con toda la información recopilada de cada corto, tomará la decisión de qué proyectar, cuándo y a quién de acuerdo con los objetivos y los destinatarios. Desde su perspectiva afirma que la aplicación de la escala EDI (Igartua y Páez, 1998) para él es una herramienta fiable para demostrar el nivel de empatía que puede tener un espectador ante la recepción de un documento audiovisual.

Volviendo a las preguntas que se formula el evaluador sobre los cortometrajes analizados, las respuestas que proporciona, ayudan a justificar su elección en función de sus objetivos e intereses.

\section{¿Es posible trabajar la educación emocional con este cortometraje?}

Por las puntuaciones registradas los cortometrajes que representan la diversidad y que aparecen en recogidos en la tabla 4, la mayoría presentan características apropiadas para trabajar la educación emocional, aunque están en función de los objetivos y de los destinatarios. Uno de los objetivos prioritarios para trabajar la educación emocional en las primeras edades es entender la situación que viven otras personas. Por este motivo, si la intención es buscar aquellos cortometrajes para trabajar la empatía en EP, es necesario seleccionar aquellos que integran a un personaje que ayuda, motiva, juega y se divierte con otro que presenta algún tipo de dificultad. El modelo de empatía que se pretende adquirir, sentir afecto vicario por otro, se enseña a través de los protagonistas de "Cuerdas", "Mi hermanito de la luna", "Broken Wings", "The present", "En tus brazos", "El cazo de Lorenzo" donde sus protagonistas apoyan en todo momento a esas personas especiales que, por circunstancias de la vida, necesitan a alguien a su lado. "Por cuatro esquinitas" de nada tiene un mensaje implícito que debe ser trabajado con los niños previamente para una correcta comprensión.

Los cortos clasificados dentro de la temática de acoso escolar y de violencia de género podrían presentar algunas dificultades a la hora de trabajar en el aula con el objetivo de promover la empatía. Por su temática, contenido y complejidad de la trama es posible que mucho alumnado no dispone de las herramientas cognitivas necesarias para entender el mensaje de los mismos (al menos en los niveles de $1^{\circ}$ a $5^{\circ}$ ).

De los cortos catalogados para trabajar los valores, algunos no cumplen con el precepto de trabajar sentimientos y competencia social: "Idiots", "Deadlines" y " $i T e$ atreves a soñar?" versan sobre contenidos empresariales como el uso de ideas con fines mercantilistas, la propagación de la obsolescencia programada en la sociedad de consumo o el uso de términos como "zona de confort" o "zona de pánico" para obtener un mayor rendimiento de la clase trabajadora. Todos ellos tienen un argumento que no guarda relación con la empatía ni los valores. Dentro de esta temática, aparecen cortos muy curiosos como "Slimtime" que refleja una sátira sobre el fascismo de la imagen o la australiana "The lost thing". Ambos films presentan protagonistas sencillos pero son sus acciones las que complican la trama y dificultan el mensaje que desean transmitir. En el caso de la primera, se puede trabajar en el aula la autoestima, el auto-concepto y, paralelamente, debatir sobre la sociedad de consumo y la importancia de la imagen personal pero no todos los niños y niñas tendrán la madurez suficiente para comprender completamente su visionado, por lo que, podrían ser interesantes para trabajar en las últimas edades de EP (10-12 años).

"The lost thing" es un cortometraje complejo y metafórico: por un lado, refleja la empatía que siente un chico por la "cosa" extraña pero después la trama se complica y no parece fácil que los niños y niñas entiendan e interpreten el significado de la misma. Por tanto, 
al igual que "Slimtime" se recomienda su uso en los últimos cursos de Educación Primaria.

El resto de películas enseñan valores como la amistad, el respeto, la importancia de aprender pero respetando el ritmo y el estilo de aprendizaje de cada persona o la solidaridad. Además, se pueden trabajar otros contenidos como el racismo, la xenofobia, la diversidad y la multiculturalidad.

\section{¿Qué aspectos positivos se aprenden?}

La diversidad de cortometrajes analizados posibilitan el abordaje de distintas temáticas desde perspectivas diferentes, al mismo tiempo que posibilita secuenciar al adquisición de conocimientos, lo que resulta muy enriquecedor. Las actividades planteadas para realizar antes de ver el corto, durante y después del visionado en la puesta en común en el aula a través de las distintas actividades, puede generar la construcción del conocimiento individual y compartido, pues el alumnado podrá debatir, argumentar, defender ideas y opinar sobre lo visto, interactuando con los demás compañeros y compañeras de tal forma que, ya de una forma implícita, están aprendiendo a empatizar y a ponerse en el lugar de otra persona.

El segundo aspecto positivo es que van a disponer, gracias a los protagonistas de los cortometrajes, de modelos de conducta con los que aprender a ayudar a los demás y rechazar o discriminar los aspectos negativos que no son agradables o no cumplan con el objetivo de empatizar.

El tercer aspecto positivo de la experiencia guarda relación con el uso de la escala EDI. En sus conclusiones, considera que puede ser útil para que cierto alumnado y familias puedan aplicarla cuando trabajen los cortos recomendados. Todos los usuarios podrán analizar si se identifican con los protagonistas e ir asimilando determinados comportamientos que les ayuden a ser mejores personas y a convertirse en seres socialmente competentes.

¿Se puede transferir lo aprendido a la vida diaria?

Sí, teniendo en cuanta que los niños y las niñas dispondrán de un modelo de valor pedagógico con el que se pueden identificar y al que podrán acudir las veces que necesiten. El hecho de poder contrastar un modelo de convivencia social con los dibujos y series animadas que ven diariamente les hará reflexionar y discriminar aquellos comportamientos negativos. Asimilar comportamientos empáticos a través de modelos audiovisuales fundamenta un buen auto concepto, ayuda a fortalecer la autoestima de forma positiva y a entenderse a sí mismos y a los demás.

\section{Conclusiones}

Esta investigación busca la reflexión del docente a partir del pretexto de buscar, seleccionar y analizar unos materiales, cortometrajes, para utilizar en su aula de EP, usando una metodología que busca la implicación del alumnado y de su familia, para trabajar la educación emocional. Esta experiencia se convierte en una oportunidad de reflexión que contribuye al enriquecimiento profesional. El maestro inicia un proceso en el que no tiene unas directrices, sino que él mismo se marca los objetivos, planifica cómo desarrollarlos y va tomando decisiones a partir de sus propias experiencias. Se encuentra solo, pues no tiene un protocolo, un catálogo que le ayude a realizar una selección de material en un universo inmenso que le proporciona internet. Recopila mucha información y es necesario filtrarla, para seleccionar lo que le interesa. Por tanto, 
exige que el docente formado, que tenga competencia digital que le ayude a hacer un uso crítico de este medio para seleccionar los cortometrajes. Identificando las fuentes y repositorios más adecuados, recopila 30 películas, que visualizará una a una, para diferenciar sus temáticas, aplicar una escala de empatía con los personajes y evaluar y clasificar por temáticas, creando un fichero que permite, de ahora en adelante, ayudar a los docentes con un nuevo recurso para trabajar los valores desde una nueva perspectiva y con una reciente metodología.

Como docente, el investigador buscó en el cortometraje un recurso atractivo, de fácil aplicación y que permitiese atender a la diversidad utilizando una metodología diferente; su importancia radica en la facilidad con la que el alumnado puede asimilar conceptos como empatía, asertividad, generosidad desde edades muy tempranas. Como protagonista de su propia práctica profesional, investiga nuevos métodos de enseñanza que ayude al conjunto de agentes implicados en la escuela a trabajar haciendo un uso de la tecnología que le permita a cada estudiante adquirir una competencia digital, crítica, constructiva y útil para construir su propio conocimiento. Sabe que es necesario despertar la curiosidad en los niños y niñas, para conseguir que el proceso de enseñanza-aprendizaje sea lo suficientemente enriquecedor y motivador para todo el alumnado, sin exclusiones.

Es aconsejable la formación del profesorado en el manejo de herramientas TIC y en que, el grado de aceptación en la implantación/integración de estas herramientas en los centros educativos es, en su mayoría, muy limitado (Méndez y Delgado, 2016). La selección de cortometrajes para enseñar los valores y las habilidades sociales parece una buena opción, siempre y cuando, se haga un estudio previo, por parte del profesor, del contenido de los mismos y, algún tipo de consulta a las familias para saber su opinión acerca de su uso en el aula. El objetivo principal de este proyecto era destacar la importancia de escoger un método y aplicar un recurso para enseñar la empatía y la inteligencia emocional en la educación. Siguiendo las palabras de Tornero, para obtener una educación plena y satisfactoria se debe "integrar un modelo educativo holístico o global que pretenda educar a la ciudadanía para la convivencia en una comunidad multicultural, desde áreas como la ecología, los derechos humanos, los valores o las actitudes" (Tornero et al. 2012). Es también necesario ser crítico a la hora de hablar de metodologías y de analizar los recursos utilizados en las mismas. La aplicación práctica del aula invertida no deja de ser una forma de repensar la enseñanza, adaptándola a nuestros días, utilizando la tecnología disponible para ello. A su favor, la etiqueta de "medida innovadora" para incentivar al alumnado a trabajar con un recurso de enorme atractivo como es el vídeo de un cortometraje de animación que enseñe la empatía. En contra, el enorme entramado organizativo que conlleva, la complejidad de su implantación, ya que, no todos los centros dispondrán de los recursos necesarios para realizarlo, así como, el enorme trabajo extra que recae sobre los hombros del profesorado y la consecuente coordinación por parte de los distintos estamentos implicados en un centro escolar; convencer, además, a todo el mundo de que una metodología es beneficiosa simplemente por usar tecnología o cambiar el orden de enseñanza es, cuando menos, utópico.

A partir de esta experiencia investigadora, es posible extraer información que le pueda ayudar a enriquecer los procesos de evaluación de los materiales, así como la toma de decisiones a cualquier docente. No obstante, ponemos en valor la importancia de contextualizar las investigaciones, por lo que de cara al futuro se optará por modelo de investigación-acción-participación.. En este sentido, resulta especialmente interesante incorporar la visión y opinión de otros participantes en el proyecto, como el alumnado y familias, con el objetivo de que ayuden a superar una toma de decisiones basada en las concepciones solas del docente. En este punto es necesario aludir a la necesidad de 
aumentar las personas implicadas en la evaluación. Sobre todo, teniendo en consideración que la flipped classroom busca la participación activa del alumnado y de su familia. Por este motivo, con el objetivo de adaptar y contextualizar los materiales a la diversidad de estudiantes y familias, será necesario abordar el proceso de selección y toma de decisiones con ellos. De esta forma, también podrá anticiparse a los posibles obstáculos que puedan surgir en el desarrollo de la experiencia.

\section{Sugerencias para el trabajo de los cortometrajes con la metodología flipped classroom}

Las actividades de la clase invertida se van a dividir en dos partes: por un lado, las actividades que realizará cada estudiante en su casa en su casa, incluyendo actividades de pre-visionado, visionado del corto y una ficha para completar mientras lo están viendo, además de cumplimentar la escala EDI, y las actividades en el aula. La siguiente tabla muestra una posible estructura de dichas actividades.

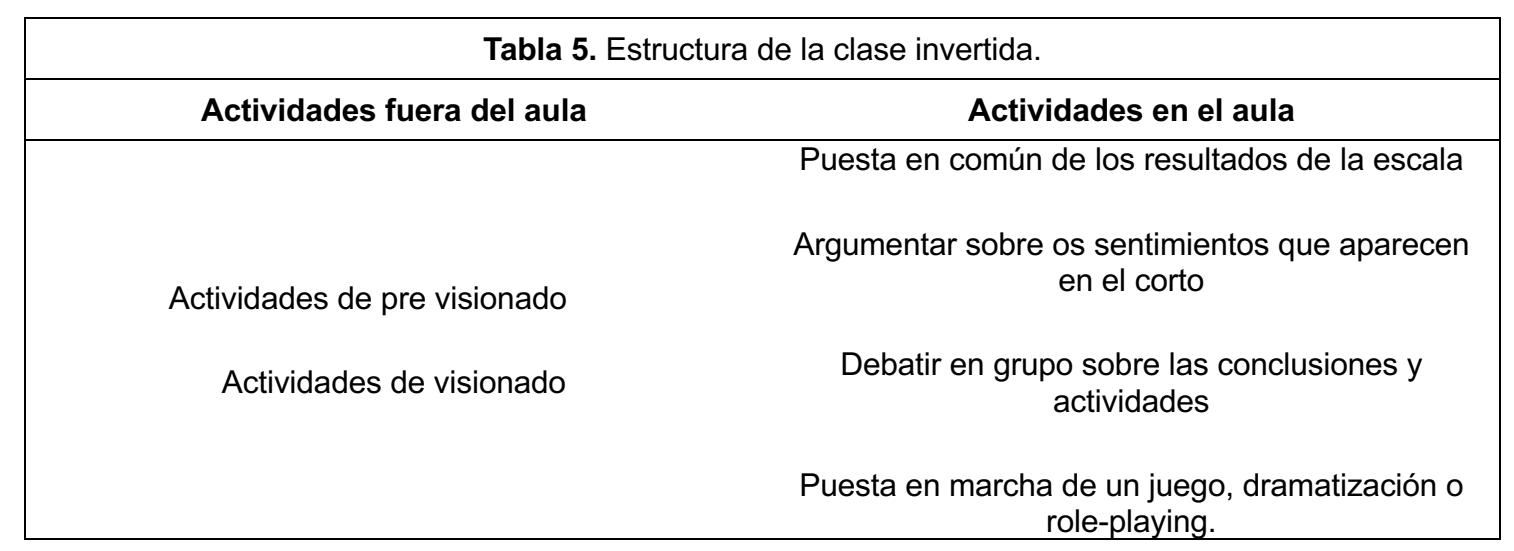

Formación de las familias para el desarrollo del método

La implicación de las familias en el proceso es fundamental. Por este motivo es necesario realizar una evaluación detallada de las características de cada unidad familiar. En esta propuesta hay que focalizar la actuación en tres aspectos fundamentales: conseguir la implicación y participación de la familia; dotar a la familia de los recursos que permitan trabajar con un material digital; que cuenten con una formación y un acompañamiento continuo para que no siente el proyecto como una carta, que tengan dificultades que no sepan solucionar y, sobre todo, que todas las familias estén en igualdad de oportunidades. Consecuentemente, es necesario realizar una formación específica en función de las necesidades individuales, pero también desarrollar iniciativas formativas con toda la comunidad. En el uso de las tecnologías, sus hijos e hijas pueden ser buenos aliados...

Es necesario identificar si cuenta con los ordenadores, móviles o tablet que puedan usar.

Si no lo tienen, el centro pondrá a disposición de las familias recursos o espacios.

Se le enseñará a utilizarlo de acuerdo con las exigencias del proyecto, que non serán muchas, debido a que sólo se trata de visualizar un vídeo.

Proporcionarles guías y materiales muy básicos que les faciliten el trabajo en casa para el trabajo con el corto. 
Resulta imprescindible, adaptar los procesos, con el objetivo que cada familia se sienta útil con sus aportaciones.

Hacerlos conscientes de sus avances y ayudarles en los obstáculos.

$1^{\circ}$ Pre visionado: Antes de ver el cortometraje, miembros de la familia, trabajarán conjuntamente para identificar emociones y sentimientos. Cada docente facilitará una serie de recursos (cuestionario, fichas de emociones, bibliografía recomendada) como preparación previa para averiguar cuáles son los sentimientos de los niños y niñas a la hora de ayudar a los demás.

$2^{a}$. Visionado. Con el visionado del cortometraje, el docente podrá profundizar a través de una serie de recursos (ficha, dibujos, escala) las potencialidades del film a la hora de despertar la empatía en sus alumnos. Se trata de trabajar de manera conjunta (docentes, alumnado, padres) como comunidad educativa preocupada por establecer vínculos emocionales positivos y desarraigar las malas conductas.

$3^{\circ}$. Post visionado. El alumnado incorporará sus aportaciones al conjunto de la clase. Cada docente puede atender a la diversidad de forma más personalizada, cumpliendo con lo establecido en la metodología flipped classroom. Se ve de nuevo el cortometraje y se da respuesta a lo que los alumnos han hecho en sus hogares con sus familias. Además, se realiza un debate y un rol-playing, evaluando los resultados obtenidos.

Sistema de evaluación del trabajo del alumnado.

Las preguntas realizadas en las actividades de pre visionado del cortometraje sirven para evaluar los conocimientos previos que tiene el alumnado sobre la empatía. Para evaluar la misma se tendrá en cuenta por lo que se establece el currículo para EP.

Existe una evaluación procesual, a través de las familias que permite corregir posibles déficits y proponer nuevas soluciones que se adapten mejor a la tipología de cada estudiante. La evaluación docente, como mecanismo de reflexión, permite hacer una actividad o una ficha contestando un cuestionario y, después, tras una reflexión personal, hacer propuestas de mejora de cara a futuras sesiones o proyectos.

\section{Referencias bibliográficas}

Aguilera-Ruiz, C., Manzano-León, A., MartínezMoreno, I., Lozano-Segura, M.C. y Yanicelli, C. C. (2017). El modelo flipped classroom. International Journal of Developmental and Educational Psychology, 4(1), 261-266. DOI: http://dx.doi.org/10.17060/ijodaep.201 7.n1.v4.1055

Alam, A., y Ahmad, M. (2018). The role of teachers' emotional intelligence in enhancing student achievement. Journal of Asia Business Studies, 12(1), 31-43. doi:10.1108/JABS-08-2015-0134

Álvarez Blanco, L., y Martínez-González, R. A. (2016). Cooperación entre las familias y los centros escolares como medida preventiva del fracaso y del riesgo de abandono escolar en adolescentes. Revista latinoamericana de educación inclusiva, 10(1), 175-192. http://dx. doi. org/10.4067/S0718-73782016000100009

Bueno i Torrens, D. (2017). Neurociencia para educadores. Barcelona: Octaedro

Chen, J. y Guo, W. (2018). Emotional intelligence can make a difference: The impact of principals' emotional intelligence on teaching strategy mediated by instructional leadership. Educational Management Administration and Leadership. 1-24 doi: 10.1177/1741143218781066 
Dafonte Gómez, A., Corbacho Valencia, J. y Miguez-González, M.I. (2017). La investigación internacional sobre flipped learning en revistas académicas. Estado de la cuestión. En Andres, S. de y otros. Actas del III Congreso Internacional de Educación Mediática y Competencia Digital. Recuperado de https://www.researchgate.net/publication/3 19465344

Del Carmen Valenzuela-Santoyo, A. y PortilloPeñuelas, S. A. (2018). Emotional intelligence in primary education and its relationship with academic performance. Revista Electrónica Educare, 22(3) doi:10.15359/ree.22-3.11

Desai, S.A., Jabeen, S. S., Abdul, W. K., y Rao, S. A. (2018). Teaching cross-cultural management: A flipped classroom approach using films. The International Journal of Management Education, 16(3), 405-431. https://doi.org/10.1016/j.ijme.2018.07.001

Fariña, F., Arce, R. y Novo, M. (2008). Neighbourhood and community factors: Effects on deviant behaviour and social competence. Spanish Journal of Psychology, 11(1), 78-84. doi:10.1017/S1138741600004133

Alonso Ferres, M., Berrocal de Luna, E. y Jiménez Sánchez, M. (2018). Estudio sobre la inteligencia emocional y los factores contextuales en estudiantes de cuarto de educación primaria de la provincia de Granada. Revista De Investigación Educativa, 36(1), doi:10.6018/rie.36.1.281441

González Fernández, G., y Carrillo Jácome, G. A. (2016). El Aprendizaje Cooperativo y la Flipped Classroom: una pareja ideal mediada por las TIC. Aularia: Revista Digital de Comunicación, 5(2), 43-48.

Gardner, H. (1995). Teoría de las Inteligencias Múltiples. La teoría en la práctica. Barcelona: Paidós Ibérica.

Hu, R., Shang, J. y Xia, Q. (2019). A study of primary school pupils' motivation, emotional intelligence and attentional control ability. In: Cheung S., Lee LK., Simonova I., Kozel T., Kwok LF. (eds) Blended Learning: Educational Innovation for Personalized Learning. Chan: Springer. Lecture Notes in Computer Science, vol 11546. doi: 10.1007/978-3-030-215620_27
Igartua Perosanz, J.J. y Paez Rovira, D. (1998). Validity and reliability of a empathy and identification with characters scale. Psicothema, 10(2), 423-436. Retrieved from www.scopus.com

Konigsberg, I. (2004). Diccionario Técnico Akal del Cine. Madrid: Akal

Marín, V. y González, I. (2006). El cine y la educación en la etapa de Primaria. Aula de Innovación, 153/154, 68-70

Mayer, J. D. y Salovey, P. (1997). What is emotional intelligence? In P. Salovey y D. J. Sluyter (Eds.). Emotional development and emotional intelligence: Educational implications, 3-34, New York: Harper Collins.http://ei.yale.edu/wpcontent/uploads/2014/02/pub219_Mayer_S alovey_1997.pdfEducativa, 153--156. 68-70.

Méndez, J. M. (2001). Aprendemos a consumir mensajes. Televisión, publicidad, prensa, radio. Huelva: Grupo Comunicar.

Méndez Garrido, J. y Delgado García, M. (2016). Las TIC en centros de Educación Primaria y Secundaria de Andalucía. Un estudio de casos a partir de buenas prácticas. Digital Education, 29, 139-165 http: // greav.ub.edu/der

Mestre, J. M., Guil, R., Lopes, P. N.; Salovey, P. y Gil-Olarte, P. (2006). Emotional intelligence and social and academic adaptation to school. Psicothema, 18, 112-117

Orón Semper, J. V. (2016). La educación emocional del adolescente entendida como integración de acuerdo con la maduración neuropsicológica. En Soler,J. L, Aparicio, L., Díaz, O., Escolano, E. y Rodríguez, A. (coords). Inteligencia Emocional $y$ Bienestar II: reflexiones, experiencias profesionales e investigaciones. Zaragoza: Ediciones, 124-139

Parrilla Latas, Á., y Moriña Díez, A. (2006). Criterios para la formación permanente del profesorado en el marco de la educación inclusiva, Revista de Educación, 339, 517539. http://hdl.handle.net/11441/66853

Pereira Domínguez, C. (2005). Los valores del cine de animación: propuestas pedagógicas para padres y educadores. Barcelona: PPU

Pulido-Acosta, F. y Herrera-Clavero, F. (2019). Prediciendo el Rendimiento Académico Infantil a través de la Inteligencia 
Emocional, Psicología Educativa, 25(1), 2330. doi: $10.5093 /$ psed2018a16

RAE (2016). Diccionario de la Real Academia de la Lengua. https://dle.rae.es/?id=DglqVCc

Rodrigo-Ruiz, D., Cejudo, J. y Pérez-González, J. C. (2019). Compendio y análisis de medidas de evaluación de la inteligencia emocional capacidad. Revista Iberoamericana De Diagnóstico $\quad$ Evaluación Psicológica, 51(2), $\quad$ 99-115. doi:10.21865/RIDEP51.2.08

Sadipour, I., Ghavam, S. E., Farrokhi, N., Assadzadeh, H. y Sameti, N. (2019). Emotional intelligence role in academic performance in smart and ordinary schools, Opcion, 35 (19), 1110-1132. Retrieved from www.scopus.com

Sánchez Gómez, Sh., Medina Moya, JL., Mendoza Pérez de Mendigurez, B., Ugarte Arena, Al. y Martínez de Albéniz Arriarán, M. (2015). Investigación acción participativa: la educación para el autocuidado del adulto maduro, un proceso dialógico y emancipador. Atención primaria: publicación oficial de la Sociedad Española de Familia y Comunitaria, 47 (9), 573-580.

Sepúlveda-Ruiz, D. P., Mayorga-Fernández, J. y Lacal, R. P. (2019). La Educación Emocional en la Educación Primaria: Un Aprendizaje para la Vida. Education Policy Analysis

Archives, 27 doi:10.14507/epaa.27.4011

Sola Martínez, T., Aznar Díaz, I., Romero Rodríguez, J. M., y Rodríguez-García, A. M. (2019). Eficacia del método Flipped Classroom en la Universidad: Meta-análisis de la producción científica de impacto. REICE. Revista Iberoamericana sobre Calidad, Eficacia y Cambio en Educación, 17 (1), 25-38. https://doi.org/10.15366/reice2019.17.1.00 2

Tornero Carrasco, M. I., Mirete Ruiz, A. B., Maquillón Sánchez, J.J. y Orcajada Sánchez, N. (2012) Metodologías y recursos para el aula multicultural. Perspectiva de maestros de educación infantil y primaria y futuros profesores de educación secundaria. Murcia: Universidad de Murcia

Torres González, J. A. (2012) Estructuras organizativas para una escuela inclusiva: promoviendo comunidades de aprendizaje. Educatio S XXI, 30 (1), 45-70
Tourón, J., Santiago, R. y Diez. A. (2014). The Flipped Classroom: Cómo convertir la escuela en un espacio de aprendizaje. España: Grupo Océano

Wirawan, H., Tamar, M., y Bellani, E. (2019). Principals' leadership styles: The role of emotional intelligence and achievement motivation. International Journal of Educational Management, 33(5), 10941105. doi:10.1108/IJEM-04-2018-0127 